\title{
The generic isogeny decomposition of the Prym Variety of a cyclic branched covering
}

\section{Theodosis Alexandrou ${ }^{1,2}$}

Received: 18 November 2021 / Accepted: 17 December 2021 / Published online: 3 January 2022

(c) The Author(s) 2022

\section{Abstract}

Let $f: S^{\prime} \longrightarrow S$ be a cyclic branched covering of smooth projective surfaces over $\mathbb{C}$ whose branch locus $\Delta \subset S$ is a smooth ample divisor. Pick a very ample complete linear system $|\mathcal{H}|$ on $S$, such that the polarized surface $(S,|\mathcal{H}|)$ is not a scroll nor has rational hyperplane sections. For the general member $[C] \in|\mathcal{H}|$ consider the $\mu_{n}$-equivariant isogeny decomposition of the Prym variety $\operatorname{Prym}\left(C^{\prime} / C\right)$ of the induced covering $f: C^{\prime}:=f^{-1}(C) \longrightarrow C$ :

$$
\operatorname{Prym}\left(C^{\prime} / C\right) \sim \prod_{d \mid n, d \neq 1} \mathcal{P}_{d}\left(C^{\prime} / C\right)
$$

We show that for the very general member $[C] \in|\mathcal{H}|$ the isogeny component $\mathcal{P}_{d}\left(C^{\prime} / C\right)$ is $\mu_{d^{-}}$ simple with $\operatorname{End}_{\mu_{d}}\left(\mathcal{P}_{d}\left(C^{\prime} / C\right)\right) \cong \mathbb{Z}\left[\zeta_{d}\right]$. In addition, for the non-ample case we reformulate the result by considering the identity component of the kernel of the map $\mathcal{P}_{d}\left(C^{\prime} / C\right) \subset$ $\operatorname{Jac}\left(C^{\prime}\right) \longrightarrow \operatorname{Alb}\left(S^{\prime}\right)$.

Keywords Jacobian variety $\cdot$ Prym variety $\cdot$ Isogeny decomposition $\cdot$ Cyclic covering

Mathematics Subject Classification $14 \mathrm{~K} 02 \cdot 14 \mathrm{~K} 12 \cdot 14 \mathrm{H} 40 \cdot 14 \mathrm{H} 10$

\section{Introduction}

For a cyclic cover $f: X \longrightarrow Y$ of smooth complex projective curves with $\operatorname{deg}(f)=n$, we fix a generator $\sigma \in \operatorname{Aut}(X / Y)$ of the automorphism group of $f$. The $\mu_{n}$-action of $X$ induces a $\mathbb{Q}$-algebra homomorphism

$$
\rho: \mathbb{Q}\left[\mu_{n}\right] \cong \mathbb{Q}[T] /\left(T^{n}-1\right) \rightarrow \operatorname{End}(\operatorname{Jac}(X)), T \mapsto \sigma^{*},
$$

Theodosis Alexandrou

alexandrou@math.uni-hannover.de

1 Mathematisches Institut, Universität Bonn, Endenicher Allee 60, Bonn 53115, Germany

2 Present Address: Institur für Algebraische Geometrie, Leibniz Universittät Hannover, Welfengarten 1, Hannover 30167, Germany 
and we define $\mathcal{P}_{d}(X / Y):=\operatorname{ker}^{0}\left(\Psi_{d}\left(\sigma^{*}\right)\right)$ for $d \mid n$, where $\Psi_{d} \in \mathbb{Z}[T]$ is the $d$-th cyclotomic polynomial. In what follows we freely use the following well-known results, which can be easily checked:

(1) $\mathcal{P}_{1}(X / Y)=\operatorname{ker}^{0}\left(\sigma^{*}-\mathrm{id}\right)=f^{*}(\operatorname{Jac}(Y)) \sim \operatorname{Jac}(Y)$

(2) The addition map $\operatorname{Jac}(Y) \times \operatorname{Prym}(X / Y) \longrightarrow \operatorname{Jac}(X),(\alpha, \beta) \mapsto f^{*}(\alpha)+\beta$ is an isogeny.

(3) Similarly, the addition map gives rise to the isogeny $\prod_{d \mid n, d \neq 1} \mathcal{P}_{d}(X / Y) \sim \operatorname{Prym}(X / Y)$.

Then, we can state the main result of this paper, which is the following:

Theorem 1.1 Let $S$ be a smooth projective surface over $\mathbb{C}$ with an ample line bundle $\mathcal{L}$. Assume $\Delta \in\left|\mathcal{L}^{\otimes n}\right|$ is smooth and consider the $n$-fold cyclic covering $f: S^{\prime} \longrightarrow S$ branched along the divisor $\Delta$. Given a very ample complete linear system $|\mathcal{H}|$ on $S$, such that $(S,|\mathcal{H}|)$ is not a scroll nor has rational hyperplane sections. Then, for the very general member $[C] \in|\mathcal{H}|$ we have that

$$
\operatorname{Prym}\left(C^{\prime} / C\right) \sim \prod_{d \mid n, d \neq 1} \mathcal{P}_{d}\left(C^{\prime} / C\right),
$$

with $\operatorname{End}_{\mu_{d}}\left(\mathcal{P}_{d}\left(C^{\prime} / C\right)\right) \cong \mathbb{Z}\left[\zeta_{d}\right]$. Especially, each $\mathcal{P}_{d}\left(C^{\prime} / C\right)$ is a $\mu_{d}$-simple abelian variety.

If we restrict to the case of double coverings, we note that the involution $\sigma$ of the covering $f$ acts as - id on $\mathcal{P}_{2}\left(C^{\prime} / C\right)=\operatorname{Prym}\left(C^{\prime} / C\right)$ and thus, $\operatorname{End}_{\mu_{2}}\left(\operatorname{Prym}\left(C^{\prime} / C\right)\right)=$ $\operatorname{End}\left(\operatorname{Prym}\left(C^{\prime} / C\right)\right)$. In particular, (1.1) can be stated as follows:

Corollary 1.2 Let $S$ be a smooth projective surface over $\mathbb{C}$ with an ample line bundle $\mathcal{L}$. Assume $\Delta \in\left|\mathcal{L}^{\otimes 2}\right|$ is smooth and consider the double covering $f: S^{\prime} \longrightarrow S$ branched along the divisor $\Delta$. Given a very ample complete linear system $|\mathcal{H}|$ on $S$, such that $(S,|\mathcal{H}|)$ is not a scroll nor has rational hyperplane sections. Then, for the very general member $[C] \in|\mathcal{H}|$ we have that

$$
\operatorname{End}\left(\operatorname{Prym}\left(C^{\prime} / C\right)\right) \cong \mathbb{Z} .
$$

The condition the line bundle $\mathcal{L}$ is ample in (1.1) implies that $\operatorname{Alb}(f): \operatorname{Alb}\left(S^{\prime}\right) \longrightarrow \operatorname{Alb}(S)$ is an isomorphism cf. page 11 and therefore the map $\mathcal{P}_{d}\left(C^{\prime} / C\right) \longrightarrow \mathrm{Alb}\left(S^{\prime}\right)$ is trivial. For the general situation one needs to consider the abelian subvariety

$$
\mathcal{R}_{d}\left(C^{\prime}, C, S^{\prime}\right):=\operatorname{ker}^{0}\left(\mathcal{P}_{d}\left(C^{\prime} / C\right) \longrightarrow \operatorname{Alb}\left(S^{\prime}\right)\right) .
$$

Then, the result can be reformulated as follows:

Theorem 1.3 Let $S$ be a smooth projective surface over $\mathbb{C}$ with a line bundle $\mathcal{L}$. Assume $\Delta \in\left|\mathcal{L}^{\otimes n}\right|$ is smooth and consider the $n$-fold cyclic covering $f: S^{\prime} \longrightarrow S$ branched along the divisor $\Delta$. Given a very ample complete linear system $|\mathcal{H}|$ on $S$, such that $(S,|\mathcal{H}|)$ is not a scroll nor has rational hyperplane sections. Then, exactly one of the following assertions holds true:

(i) For the general member $[C] \in|\mathcal{H}|$ we have that $\mathcal{R}_{d}\left(C^{\prime}, C, S^{\prime}\right)=0$.

(ii) For the very general member $[C] \in|\mathcal{H}|$ we have that $\operatorname{End}_{\mu_{d}}\left(\mathcal{R}_{d}\left(C^{\prime}, C, S^{\prime}\right)\right) \cong \mathbb{Z}\left[\zeta_{d}\right]$.

In this paper we present a complete proof for the above results, inspired by Ciliberto and Van der Geer's approach in [3]. We note that this method does not capture the étale situation, cf. (3.2), (3.3) and (3.4). In addition, if we rephrase the statement for $n>2$ by requiring simplicity instead of $\mu_{d}$-simplicity to the isogeny components, we observe that this method cannot be adopted. Namely, the abelian variety $B$ in (3.4) cannot be chosen in general to 
be $\mu_{d}$-invariant and for this reason the last combinatorial argument in (3.4) fails. Lastly, a result due to Ortega and Lange, cf. [6] may be used to find counter-example for the case the covering $f$ is étale of degree 7 .

Notations and Conventions. For $n \in \mathbb{N}, \mu_{n}$ is the constant group scheme over $\mathbb{C}$, which is associated to the abstract group $\mathbb{Z} / n \mathbb{Z}$. The symbol $\zeta_{n}$ stands for a primitive $n$-th root of unity. If $A$ is an abelian variety over $\mathbb{C}$, which is endowed with a $\mu_{n}$-action, then $\operatorname{End}_{\mu_{n}}(A)$ is the ring of $\mu_{n}$-equivariant endomorphisms of $A$. A very general point of a given variety $X$ is a closed point $x \in X$, that lies in the complement of a countable union of nowhere dense closed subvarieties.

\section{Preliminaries}

In this section, we state some well-known results, which are needed later.

Proposition 2.1 Let $\pi: \mathcal{A} \longrightarrow S$ be a projective abelian scheme over a Noetherian base $S$. Then, the endomorphism functor of $\mathcal{A}$ over $S$ is representable by an $S$-scheme End Al $_{S}$, which is a disjoint union of projective and unramified $S$-schemes.

Proof This is well-known, cf. [4, pp. 133].

The following proposition relates the correspondences on $C \times C$ with the endomorphisms of the Jacobian $\operatorname{Jac}(C)$.

Proposition 2.2 Let $\pi: \mathcal{X} \longrightarrow S$ be a projective smooth morphism over a Noetherian base $S$, whose fibres are geometrically integral curves. Furthermore, assume that the morphism $\pi$ admits a section, i.e. $\mathcal{X}(S) \neq \emptyset$. Then, there is a natural and functorial isomorphism

$$
\operatorname{Corr}_{S}(\mathcal{X}):=\operatorname{Pic}\left(\mathcal{X} \times{ }_{S} \mathcal{X}\right) /\left(\operatorname{pr}_{1}\right)^{*} \operatorname{Pic}(\mathcal{X}) \otimes\left(\operatorname{pr}_{2}\right)^{*} \operatorname{Pic}(\mathcal{X}) \cong \operatorname{End}_{S}\left(\operatorname{Pic}_{\mathcal{X} / S}^{0}\right) .
$$

Proof Consider the commutative diagram:

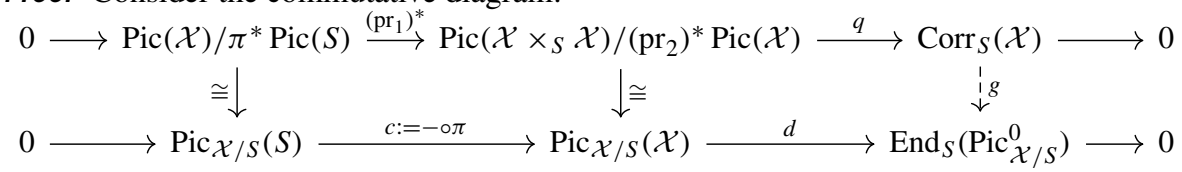

The first row is clearly exact: Indeed, the relative Picard functor is an fppf-sheaf, cf. [13, Tag 021L], [5, Thm. 2.5] and thus, the restriction map $\left(\mathrm{pr}_{1}\right)^{*}$ is injective. Furthermore, the map $q$ is just the cokernel of $\left(\mathrm{pr}_{1}\right)^{*}$. Next, we give the definition of the map $d$. Fix $x \in \mathcal{X}(S)$ and let $\phi: \mathcal{X} \longrightarrow \operatorname{Pic} \mathcal{X} / S$ be any $S$-morphism. Then, $d \phi$ is the unique endomorphism of $\operatorname{Pic}_{\mathcal{X} / S}^{0}$, making the diagram below commutative.

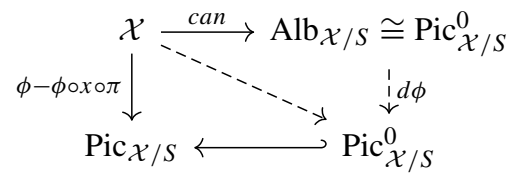

Note that under our assumptions the Albanese map can: $\mathcal{X} \longrightarrow \mathrm{Alb}_{\mathcal{X} / S}$ exists and has the desired universal property, cf. [1, Thm. 2.17], [1, Rem. 2.19] and [[8], Thm. 10.2]. Moreover, the construction of the map $d$ indicates that $d$ is surjective and also that the second row in the diagram above is exact at the middle. Now, the existence of $g$ and the fact that it is an isomorphism are clear, since the first two vertical maps are isomorphisms by [5, Thm. 4.8] and [5, Thm. 2.5]. 
The following proposition is well-known.

Proposition 2.3 Suppose that the polarized surface $(S,|\mathcal{H}|)$ is not a scroll nor has rational hyperplane sections. Then, the following assertions hold true:

(i) The discriminant divisor $\mathcal{D}$ is irreducible and has codimension one in $|\mathcal{H}|$, i.e. $\mathcal{D}$ is a prime divisor of $|\mathcal{H}|$.

(ii) The general curve $[C] \in \mathcal{D}$ is irreducible and has a single ordinary double point as its only singularity.

Proof Cf. [3, Lem. 3.1].

We close this section by introducing the $\mu_{n}$-equivariant isogeny decomposition in (1.1). Let $f: C^{\prime} \longrightarrow C$ be a cyclic branched covering of smooth complex projective curves with $\operatorname{deg}(f)=n$ and let $\sigma$ stand for a generator of the Galois group of $f$. The $\mu_{n}$-action on $C^{\prime}$ induces an action on $\operatorname{Jac}\left(C^{\prime}\right)$ and thus, it defines a $\mathbb{Q}$-algebra homomorphism

$$
\rho: \mathbb{Q}\left[\mu_{n}\right] \cong \mathbb{Q}[T] /\left(T^{n}-1\right) \longrightarrow \operatorname{End}^{0}\left(\operatorname{Jac}\left(C^{\prime}\right)\right), T \mapsto \sigma^{*} .
$$

For any divisor $d \mid n$, we define $\mathcal{P}_{d}\left(C^{\prime} / C\right):=\operatorname{ker}^{0}\left(\Psi_{d}\left(\sigma^{*}\right)\right)$, where $\Psi_{d}(T) \in \mathbb{Z}[T]$ is the $d$-th cyclotomic polynomial. Then, the addition map

$$
\mu: \prod_{d \mid n} \mathcal{P}_{d}\left(C^{\prime} / C\right) \longrightarrow \operatorname{Jac}\left(C^{\prime}\right)
$$

is a $\mu_{n}$-equivariant isogeny. Lange and Recillas [7] have stated and proved the relation between $\mathbb{Q}$-representations and the $G$-equivariant isogeny decomposition of an abelian variety with $G$-action, in terms of the finite group $G$ involved, cf. [7, Thm. 2.2]. The $\mu_{n^{-}}$ equivariant isogeny decomposition of $\operatorname{Jac}\left(C^{\prime}\right)$ given above is in fact identical with the one introduced by Lange and Recillas [7]. This can be seen for example by using [2, Rem. 5.5] and [2, Cor. 5.7]. Moreover, we also note that the isogeny components $\mathcal{P}_{d}\left(C^{\prime} / C\right)$ are non-trivial as long as the genus $g(C) \geq 1$, cf. [7, Thm. 3.1], [11, Thm. 5.12] and [11, Thm. 5.13].

\section{Reduction to the generic fibre}

Let $S$ be a smooth projective surface over $\mathbb{C}$ with an ample line bundle $\mathcal{L}$. Assume $\Delta \in\left|\mathcal{L}^{\otimes n}\right|$ is smooth and consider the $n$-fold cyclic covering $f: S^{\prime} \longrightarrow S$ branched along the divisor $\Delta$. Furthermore, fix a very ample complete linear system $|\mathcal{H}|$ on $S$, such that the polarized surface $(S,|\mathcal{H}|)$ is not a scroll nor has rational hyperplane sections. In this section we reduce the proof of Theorem 1.1 to showing that $\mathcal{P}_{d}\left(C_{\eta}^{\prime} / C_{\eta}\right)$ is a $\mu_{d}$-simple abelian variety, where $\left[C_{\eta}\right]$ is the generic member of $|\mathcal{H}|$.

Let $x \in S$ be a closed point of $S$. We denote by $|\mathcal{H}|_{x}$ the linear system of hyperplane sections in $|\mathcal{H}|$ passing through $x$. In the following we impose restrictions on the point $x$, i.e. $x \in S$ will be taken from some appropriate non-empty open subset of $S$.

Let $g: \mathcal{X} \subset S \times|\mathcal{H}|_{x} \longrightarrow|\mathcal{H}|_{x}$ denote the universal family of hyperplane sections and $h: \mathcal{Y} \subset S^{\prime} \times|\mathcal{H}|_{x} \longrightarrow|\mathcal{H}|_{x}$ its pullback to $S^{\prime}$, i.e. $\mathcal{Y}:=\mathcal{X} \times{ }_{S} S^{\prime}$. Note that over the non-empty open subset $U \subset|\mathcal{H}|_{x}$ of smooth curves which intersect the branch locus $\Delta$ transversally both $g$ and $h$ are smooth families of curves having a section. The latter allows us to consider their families of Jacobians over $U$, which we denote by $p: \operatorname{Pic}_{\mathcal{X} / U}^{0} \longrightarrow U$ and $q: \operatorname{Pic}_{\mathcal{Y} / U}^{0} \longrightarrow U$, respectively. 
A generator $\sigma: S^{\prime} \longrightarrow S^{\prime}$ of the Galois group of the covering $f$ induces an automorphism of $\mathcal{Y}$ over $U$ and thus, an automorphism $\sigma^{*}: \operatorname{Pic}_{\mathcal{Y} / U}^{0} \longrightarrow \operatorname{Pic}_{\mathcal{Y} / U}^{0}$. We define

$$
\mathcal{P}_{d}:=\operatorname{ker}^{0}\left(\Psi_{d}\left(\sigma^{*}\right)\right) \text { for any divisor } d \mid n .
$$

Then, $\varphi_{d}: \mathcal{P}_{d} \longrightarrow U$ is an abelian fibration with fibres $\left(\mathcal{P}_{d}\right)_{[C]}=\mathcal{P}_{d}\left(C^{\prime} / C\right)$ for $[C] \in U$.

As a first step we use the representability of the endomorphism functor of abelian schemes cf. (2.1) to reduce the proof of Theorem 1.1 to showing that $\operatorname{End}_{\mu_{d}}\left(\left(\mathcal{P}_{d}\right)_{\bar{\eta}}\right) \cong \mathbb{Z}\left[\zeta_{d}\right]$, where $\bar{\eta}$ is a fixed geometric generic point of $|\mathcal{H}|_{x}$. The proof of this is standard and so we omit it.

Lemma 3.1 Assume that $\operatorname{End}_{\mu_{d}}\left(\left(\mathcal{P}_{d}\right)_{\bar{\eta}}\right) \cong \mathbb{Z}\left[\zeta_{d}\right]$. Then, for the very general member $[C] \in$ $U$, one has that $\operatorname{End}_{\mu_{d}}\left(\left(\mathcal{P}_{d}\right)_{[C]}\right) \cong \mathbb{Z}\left[\zeta_{d}\right]$.

Let $[C] \in|\mathcal{H}|_{x}$ be an irreducible member with a single ordinary double point as its only singularity and intersecting the branch locus $\Delta$ transversally. Then, $C^{\prime}:=f^{-1}(C)$ is irreducible and has $n$ ordinary double points as its only singularities. In this case the group variety $\mathcal{P}_{d}\left(C^{\prime} / C\right)$ is semi-abelian. In particular, the result is the following:

Lemma 3.2 For an irreducible member $[C] \in|\mathcal{H}|_{x}$ with a single ordinary double point as its only singularity and intersecting the branch locus $\Delta$ transversally, there is an exact sequence:

$$
0 \longrightarrow \mathbb{G}_{m}^{\varphi(d)} \longleftrightarrow \mathcal{P}_{d}\left(C^{\prime} / C\right) \longrightarrow \mathcal{P}_{d}\left(\tilde{C}^{\prime} / \tilde{C}\right) \longrightarrow 0,
$$

where $v: \tilde{C} \longrightarrow C$ is the normalisation map and $\varphi(d)$ is the Euler's totient function.

Proof We have a commutative diagram

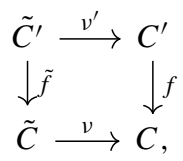

where $\tilde{f}$ is the cyclic covering branched along the divisor $\left.v^{*} \Delta\right|_{C} \in\left|v^{*} \mathcal{L}\right|_{C}^{\otimes n} \mid$ and $v^{\prime}$ is the normalisation of $C^{\prime}$. Fix a generator $\sigma$ of $\operatorname{Aut}\left(C^{\prime} / C\right)$ and let $\tilde{\sigma}$ be the corresponding generator of $\operatorname{Aut}\left(\tilde{C}^{\prime} / \tilde{C}\right)$, i.e. the one for which the diagram below commutes

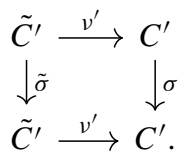

Let $\left\{y, \sigma(y), \sigma^{2}(y), \ldots, \sigma^{n-1}(y)\right\}$ be the set of ordinary double points of $C^{\prime}$. Then, we find a commutative diagram with exact rows and columns

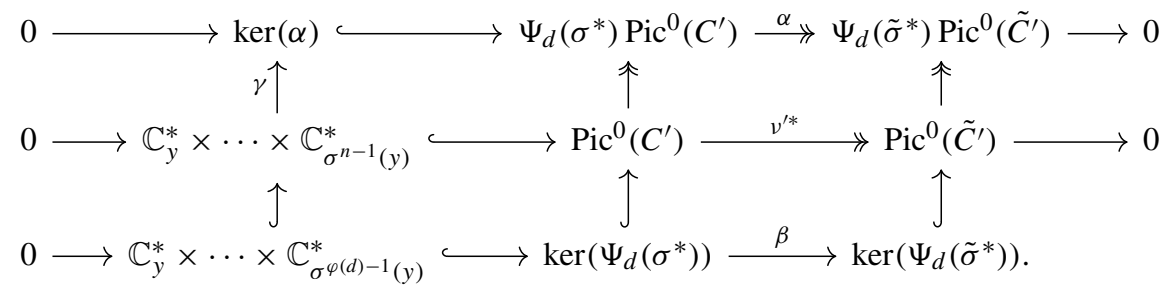


We show that $\beta$ induces a surjection $\mathcal{P}_{d}\left(C^{\prime} / C\right)=\operatorname{ker}^{0}\left(\Psi_{d}\left(\sigma^{*}\right)\right) \rightarrow \mathcal{P}_{d}\left(\tilde{C}^{\prime} / \tilde{C}\right)=$ $\operatorname{ker}^{0}\left(\Psi_{d}\left(\tilde{\sigma}^{*}\right)\right)$. Indeed, by Snake lemma we have the exact sequence

$$
\operatorname{ker}\left(\Psi_{d}\left(\sigma^{*}\right)\right) \longrightarrow \operatorname{ker}\left(\Psi_{d}\left(\tilde{\sigma}^{*}\right)\right) \longrightarrow \operatorname{coker}(\gamma) \longrightarrow 0 .
$$

Note that $\operatorname{coker}(\gamma)$ is an affine algebraic group, as it is the quotient of a commutative affine algebraic group by an algebraic subgroup. Since $\operatorname{ker}\left(\Psi_{d}\left(\tilde{\sigma}^{*}\right)\right)$ is a projective variety and the last arrow in the above sequence is surjective, [14, Cor. 12.67] shows that $\operatorname{coker}(\gamma)$ is finite. The latter provides the surjectivity of the map $\operatorname{ker}^{0}\left(\Psi_{d}\left(\sigma^{*}\right)\right) \longrightarrow \mathcal{P}_{d}\left(\tilde{C}^{\prime} / \tilde{C}\right)=$ $\operatorname{ker}^{0}\left(\Psi_{d}\left(\tilde{\sigma}^{*}\right)\right)$, as claimed.

We are now in the position to prove the following:

Proposition 3.3 The abelian variety $\left(\mathcal{P}_{d}\right)_{\bar{\eta}}$ is $\mu_{d}$-simple if and only if $\operatorname{End}_{\mu_{d}}\left(\left(\mathcal{P}_{d}\right)_{\bar{\eta}}\right) \cong \mathbb{Z}\left[\zeta_{d}\right]$.

Proof The one direction is clear: Indeed, if $\operatorname{End}_{\mu_{d}}\left(\left(\mathcal{P}_{d}\right)_{\bar{\eta}}\right) \cong \mathbb{Z}\left[\zeta_{d}\right]$, then every non-zero $\mu_{d}$-equivariant endomorphism of $\left(\mathcal{P}_{d}\right)_{\bar{\eta}}$ is an isogeny and thus, $\left(\mathcal{P}_{d}\right)_{\bar{\eta}}$ is a $\mu_{d}$-simple abelian variety. Conversely, assume that $\left(\mathcal{P}_{d}\right)_{\bar{\eta}}$ is $\mu_{d}$-simple. We divide the proof into steps.

Step 1. There is a closed subscheme $\operatorname{End}_{\mathcal{P}_{d} / U}^{\mu_{d}}(0) \subset \operatorname{End}_{\mathcal{P}_{d} / U}^{\mu_{d}}$ whose points parametrise the $\mu_{d}$-equivariant endomorphisms of $\mathcal{P}_{d}$, which are not isogenies, i.e. the ones, which are of degree 0 .

Proof of Step 1 Observe that the functor of $\mu_{d}$-equivariant endomorphisms of $\mathcal{P}_{d}$ denoted by End $_{\mathcal{P}_{d} / U}^{\mu_{d}}$ is representable by a closed subscheme of $\operatorname{End}_{\mathcal{P}_{d} / U}$, since the equivariant condition is closed. It follows that we have a universal endomorphism $\alpha$, such that every other $\mu_{d^{-}}$ equivariant endomorphism of $\mathcal{P}_{d}$ over some scheme $T$ is obtained by pulling-back $\alpha$ along a morphism $T \longrightarrow \operatorname{End}_{\mathcal{P}_{d} / U}^{\mu_{d}}$. By [14, Prop. 12.93] the set

$$
\mathcal{V}:=\left\{x \in \operatorname{End}_{\mathcal{P}_{d} / U}^{\mu_{d}} \mid \alpha_{x}:=\alpha \times \operatorname{id}_{\kappa(x)} \text { is an isogeny }\right\}
$$

is open. Therefore, $\operatorname{End}_{\mathcal{P}_{d} / U}^{\mu_{d}}(0):=\operatorname{End}_{\mathcal{P}_{d} / U}^{\mu_{d}} \backslash \mathcal{V}$ with the reduced induced closed subscheme structure has the desired property.

Step 2. The fibre $\left(\mathcal{P}_{d}\right)_{[C]}$ for the very general member $[C] \in|\mathcal{H}|_{x}$ is a $\mu_{d}$-absolutely simple abelian variety.

Proof of Step 2 Recall that the $U$-scheme $\operatorname{End}_{\mathcal{P}_{d} / U}^{\mu_{d}}(0)$ is unramified cf. (2.1). It follows that a geometric fibre of this $U$-scheme is a disjoint union of points, corresponding to the $\mu_{d^{-}}$ equivariant endomorphisms of $\mathcal{P}_{d}$, which are not isogenies cf. Step 1 . Since $\left(\mathcal{P}_{d}\right)_{\bar{\eta}}$ is a $\mu_{d}$-simple abelian variety, the only $\mu_{d}$-equivariant endomorphism of $\left(\mathcal{P}_{d}\right)_{\bar{\eta}}$, that is not an isogeny is the zero-morphism. In particular, this means that the geometric generic fibre of the $U$-scheme $\operatorname{End}_{\mathcal{P}_{d} / U}^{\mu_{d}}(0)$ is connected and therefore, we can determine countably many non-empty open subsets $U_{i} \subset U$, such that the $U$-scheme $\operatorname{End}_{\mathcal{P}_{d} / U}^{\mu_{d}}(0)$ has (geometrically) connected fibres for all points lying in the intersection of the $U_{i}$ 's, cf. [13, Tag 055C]. Thus, for the very general member $[C] \in|\mathcal{H}|_{x}$, the only $\mu_{d}$-equivariant endomorphism of $\left(\mathcal{P}_{d}\right)_{[C]}$, which is not an isogeny is the zero-morphism. The latter is equivalent to the $\mu_{d}$-simplicity of $\left(\mathcal{P}_{d}\right)_{[C]}$, proving the claim.

Pick a Lefschetz pencil $\left(C_{t}\right)_{t \in \mathbb{P}^{1}} \subset|\mathcal{H}|_{x}$. We may assume that all its singular members are irreducible and intersect the branch locus $\Delta$ transversally, cf. (2.3).

Step 3. Given a Lefschetz pencil $\left(C_{t}\right)_{t \in \mathbb{P}^{1}}$ as above, we construct a homomorphism:

$$
\rho: \operatorname{End}_{\mu_{d}}\left(\left(\mathcal{P}_{d}\right)_{\bar{\mu}}\right) \longrightarrow \operatorname{End}\left(\mathbb{G}_{m}^{\varphi(d)}\right),
$$

where $\bar{\mu}$ is a fixed geometric generic point of $\mathbb{P}^{1}$. 
Proof of Step 3 Since the endomorphism ring of any abelian variety is finitely generated, cf. [[9], Thm. 12.5], we find a finite field extension $L \supset \kappa(\mu)$, such that every endomorphism of $\mathcal{P}_{d}$ over $\kappa(\bar{\mu})$ is defined over $L$, i.e. $\operatorname{End}\left(\left(\mathcal{P}_{d}\right)_{\bar{\mu}}\right)=\operatorname{End}\left(\left(\mathcal{P}_{d}\right)_{L}\right)$. Consider the smooth projective model $E$ of $L$ together with the morphism $E \longrightarrow \mathbb{P}^{1}$ induced by this field extension and fix a closed point $y \in E$ lying over a point of the pencil that corresponds to a nodal curve. The map $\rho: \operatorname{End}_{\mu_{d}}\left(\left(\mathcal{P}_{d}\right)_{\bar{\mu}}\right) \longrightarrow \operatorname{End}\left(\mathbb{G}_{m}^{\varphi(d)}\right)$ is constructed as follows: Let $f \in$ $\operatorname{End}_{\mu_{d}}\left(\left(\mathcal{P}_{d}\right)_{L}\right)$. Then, $f$ extends to an endomorphism over the local ring $R$ of $E$ at the point $y$, cf. [12, Prop. 7.4.3]. The restriction of the first projection of $\mathcal{P}_{d} \times{ }_{R} \mathcal{P}_{d}$ to the graph of $f$ is an isomorphism. We set $\alpha:=\left.\operatorname{pr}_{1}\right|_{\left(\Gamma_{f}\right)_{y}}$. By pulling back $\alpha$ along $\mathbb{G}_{m}^{\varphi(d)} \hookrightarrow\left(\mathcal{P}_{d}\right)_{y}$, we get an isomorphism $\alpha: \alpha^{-1}\left(\mathbb{G}_{m}^{\varphi(d)}\right) \longrightarrow \mathbb{G}_{m}^{\varphi(d)}$. We claim that $\alpha^{-1}$ is the graph of a homomorphism $\mathbb{G}_{m}^{\varphi(d)} \longrightarrow \mathbb{G}_{m}^{\varphi(d)}$. Indeed, it suffices to show that $\operatorname{pr}_{2}\left(\alpha^{-1}\left(\mathbb{G}_{m}^{\varphi(d)}\right)\right) \subset \mathbb{G}_{m}^{\varphi(d)}$. To see this, observe that the composite

$$
\mathbb{G}_{m}^{\varphi(d)} \stackrel{\cong}{\longrightarrow} \alpha^{-1}\left(\mathbb{G}_{m}^{\varphi(d)}\right) \subset\left(\Gamma_{f}\right)_{y} \stackrel{\mathrm{pr}_{2}}{\longrightarrow}\left(\mathcal{P}_{d}\right)_{y} \longrightarrow \mathcal{P}_{d}\left(\tilde{C}_{y}^{\prime} / \tilde{C}_{y}\right)
$$

is the zero map by [[9], Cor. 3.9] and hence, $\left.\operatorname{pr}_{2}\right|_{\mathbb{G}_{m}^{\varphi(d)}}$ factors through the kernel of $\left(\mathcal{P}_{d}\right)_{y} \longrightarrow$ $\mathcal{P}_{d}\left({\tilde{C_{y}^{\prime}}}_{y} / \tilde{C}_{y}\right)$ which is $\mathbb{G}_{m}^{\varphi(d)}$. Finally, we define $\rho(f)$ to be this endomorphism of $\mathbb{G}_{m}^{\varphi(d)}$. One checks that $\rho$ is a homomorphism of rings.

Conclusion Eventually, we are in the position to complete the proof. Suppose $\operatorname{End}_{\mu_{d}}\left(\left(\mathcal{P}_{d}\right)_{\bar{\eta}}\right) \neq$ $\mathbb{Z}\left[\zeta_{d}\right]$ and choose a $\mu_{d}$-equivariant endomorphism $f$ not in $\mathbb{Z}\left[\zeta_{d}\right]$. The endomorphism $f$ can be described as a $\kappa(\bar{\eta})$-point of $\operatorname{End}_{\mathcal{P}_{d} / U}^{\mu_{d}}$ and we let $Z \subset \operatorname{End}_{\mathcal{P}_{d} / U}^{\mu_{d}}$ be the irreducible component containing this point. Then, the generic point $\theta \in Z$ corresponds to a $\mu_{d}$-equivariant endomorphism not in $\mathbb{Z}\left[\zeta_{d}\right]$. Consider the finite set

$$
\Gamma:=\left\{n:=\left(n_{0}, n_{1}, \ldots, n_{\varphi(d)-1}\right) \in \mathbb{Z}^{\varphi(d)} \mid \operatorname{im}\left([n]^{1}\right) \cap Z \neq \emptyset\right\} .
$$

Each $\operatorname{im}([n]) \cap Z$ is a proper closed subset of $Z$. Setting ${ }^{1}$

$$
Z_{n}:=\pi(\operatorname{im}([n]) \cap Z),
$$

for $n \in \Gamma$, we get finitely many nowhere dense closed subsets of $U$, such that for every point $u \in U \backslash \bigcup_{n \in \Gamma} Z_{n}$ the fibre $\pi^{-1}(u)$ contains a point, which is not in $\mathbb{Z}\left[\zeta_{d}\right]$. We can choose a Lefschetz pencil as above, such that $\left(\mathcal{P}_{d}\right)_{\bar{\mu}}$ is $\mu_{d}$-simple, cf. Step 2 and $\operatorname{End}_{\mu_{d}}\left(\left(\mathcal{P}_{d}\right)_{\bar{\mu}}\right) \neq$ $\mathbb{Z}\left[\zeta_{d}\right]$. By Step 3 this leads to a contradiction. Indeed, using that every non-zero element of $\operatorname{End}_{\mu_{d}}\left(\left(\mathcal{P}_{d}\right)_{\bar{\mu}}\right)$ is invertible in $\operatorname{End}_{\mu_{d}}\left(\left(\mathcal{P}_{d}\right)_{\bar{\mu}}\right) \otimes \mathbb{Q}$, it is readily checked that the composition of the map $\rho$ constructed in Step 3 with $\psi:=\operatorname{pr}_{1} \circ-: \operatorname{End}\left(\mathbb{G}_{m}^{\varphi(\delta)}\right) \longrightarrow \operatorname{Hom}\left(\mathbb{G}_{m}^{\varphi(\delta)}, \mathbb{G}_{m}\right) \cong$ $\mathbb{Z}^{\varphi(\delta)}$ is injective. It follows that $\operatorname{End}_{\mu_{d}}\left(\left(\mathcal{P}_{d}\right)_{\bar{\mu}}\right) \otimes \mathbb{Q} \cong \mathbb{Q}\left(\zeta_{d}\right)$. Since $\mathbb{Z}\left[\zeta_{d}\right]$ is a maximal order in $\mathbb{Q}\left(\zeta_{d}\right)$, we also obtain $\operatorname{End}_{\mu_{d}}\left(\left(\mathcal{P}_{d}\right)_{\bar{\mu}}\right) \cong \mathbb{Z}\left[\zeta_{d}\right]$. The proof is complete.

The next lemma consists of the final reduction step.

Lemma 3.4 The abelian variety $\left(\mathcal{P}_{d}\right)_{\eta}$ is $\mu_{d}$-simple if and only if it is $\mu_{d}$-absolutely simple.

Proof Clearly, if $\left(\mathcal{P}_{d}\right)_{\eta}$ is $\mu_{d}$-absolutely simple, then it is $\mu_{d}$-simple. Conversely, assume that $\left(\mathcal{P}_{d}\right)_{\eta}$ is $\mu_{d}$-simple but not $\mu_{d}$-absolutely simple. Then, there is a finite field extension $L \supset \kappa(\eta)$ and a non-zero and proper $\mu_{d}$-simple abelian subvariety $B$ of $\left(\mathcal{P}_{d}\right)_{L}$, such that $\left(\mathcal{P}_{d}\right)_{L}$ can be written up to isogeny as a product $\prod B^{\tau}$, where $B^{\tau}$ stands for a Galois conjugate of $B$ and $\tau$ runs through a finite subset $J \subset \operatorname{Gal}(L / \kappa(\eta))$ of cardinality greater equal to 2 . The field extension $L \supset \kappa(\eta)$ gives rise to a morphism $g: U^{\prime} \longrightarrow U$, which we may assume

$\overline{1_{[n]:=n_{0} \mathrm{id}+n_{1} \sigma^{*}+n_{2}\left(\sigma^{*}\right)^{2}}}+\cdots+n_{\varphi(d)-1}\left(\sigma^{*}\right)^{\varphi(d)-1}$. 
is étale. For $\tau \in J$, we let $\varphi_{\tau}$ be the endomorphism of $\left(\mathcal{P}_{d}\right)_{L}$ whose image is $B^{\tau}$. More explicitly, $\varphi_{\tau}$ is given by

$$
\left(\mathcal{P}_{d}\right)_{L} \stackrel{\sim}{\longrightarrow} \prod B^{\tau} \stackrel{\text { proj }}{\longrightarrow} B^{\tau} \subset\left(\mathcal{P}_{d}\right)_{L}
$$

Pick a Lefschetz pencil $\left(C_{t}\right)_{t \in \mathbb{P}^{1}}$, such that its singular members are irreducible and intersect the branch locus $\Delta$ transversally. Let $X$ be any irreducible component of $g^{-1}\left(\mathbb{P}^{1} \cap U\right)$. Then, $X$ dominates $\mathbb{P}^{1} \cap U$ and if $\theta \in X$ is its generic point, then each $\varphi_{\tau}$ determines an endomorphism of $\mathcal{P}_{d}$ over $\theta$, e.g. using the Néron mapping property, such that if $B^{\tau}:=\operatorname{im}\left(\varphi_{\tau}\right)$, then $\prod B^{\tau} \sim\left(\mathcal{P}_{d}\right)_{\theta}$. Let $\bar{X}$ be a smooth compactification of $X$ and $\bar{X} \longrightarrow \mathbb{P}^{1}$ the extension of $g: X \longrightarrow \mathbb{P}^{1} \cap U$. Fix a point $y \in \bar{X}$ lying over a point of the pencil which corresponds to a nodal curve and consider the local ring $R$ of $\bar{X}$ at $y$. Since $\mathcal{P}_{d}$ admits a semi-abelian reduction over $R$, cf. (3.2) the same is true for all $B^{\tau}$, cf. [12, Cor. 7.1.6]. We denote by $\tilde{B}^{\tau}$ the identity component of the Néron model of $B^{\tau}$. Then, the isogeny of the generic fibre extends to an isogeny $\prod \tilde{B}^{\tau} \sim \mathcal{P}_{d}$ over $R$, cf. [12, Prop. 7.3.6]. Since $\left(\mathcal{P}_{d}\right)_{y}$ is an extension of an abelian variety by a torus of rank $\varphi(d)$, cf. (3.2), it follows that the toric part of $\tilde{B}_{y}^{\tau}$ has $\operatorname{rank} \delta, 1 \leq \delta \leq \varphi(d)$, such that $\delta|J|=\varphi(d)$. As in Step 3, one constructs a homomorphism $\rho_{\tau}: \operatorname{End}_{\mu_{d}}\left(B^{\tau}\right) \longrightarrow \operatorname{End}\left(\mathbb{G}_{m}^{\delta}\right)$. Since the restriction of $\psi \circ \rho_{\tau}$ to $\mathbb{Z}\left[\zeta_{d}\right] \subset \operatorname{End}_{\mu_{d}}\left(B^{\tau}\right)$ is injective, where $\psi:=\operatorname{pr}_{1} \circ-: \operatorname{End}\left(\mathbb{G}_{m}^{\delta}\right) \longrightarrow \operatorname{Hom}\left(\mathbb{G}_{m}^{\delta}, \mathbb{G}_{m}\right) \cong \mathbb{Z}^{\delta}$ and $\mathbb{Z}\left[\zeta_{d}\right]$ has rank $\varphi(d)$ as a free abelian group, we conclude that $\delta=\varphi(d)$. But then $|J|=1$, which is absurd.

\section{The Proof of Theorem 1.1}

According to the results of Sect. 3, our task to prove Theorem 1.1 is reduced to showing $\left(\mathcal{P}_{d}\right)_{\eta}$ is a $\mu_{d}$-simple abelian variety. Recall, that we have an isogeny

$$
\operatorname{Jac}\left(C_{\eta}^{\prime}\right) \sim \operatorname{Jac}\left(C_{\eta}\right) \times \prod_{d \mid n, d \neq 1}\left(\mathcal{P}_{d}\right)_{\eta} .
$$

Given a non-zero endomorphism $\varepsilon \in \operatorname{End}_{\mu_{d}}\left(\left(\mathcal{P}_{d}\right)_{\eta}\right)$. Then, by considering the composite

$$
\varepsilon^{\prime}: \operatorname{Jac}\left(C_{\eta}^{\prime}\right) \stackrel{\sim}{\longrightarrow} \operatorname{Jac}\left(C_{\eta}\right) \times \prod_{d \mid n, d \neq 1}\left(\mathcal{P}_{d}\right)_{\eta} \stackrel{\mathrm{pr}_{d}}{\longrightarrow}\left(\mathcal{P}_{d}\right)_{\eta} \stackrel{\varepsilon}{\longrightarrow}\left(\mathcal{P}_{d}\right)_{\eta} \hookrightarrow \operatorname{Jac}\left(C_{\eta}^{\prime}\right),
$$

we get an endomorphism of $\operatorname{Jac}\left(C_{\eta}^{\prime}\right)$ whose restriction to $\left(\mathcal{P}_{d}\right)_{\eta}$ is simply $\varepsilon \circ[n]$. Hence, it suffices to show that that the restriction of $\varepsilon^{\prime}$ to $\left(\mathcal{P}_{d}\right)_{\eta}$ lies in $\mathbb{Z}\left[\zeta_{d}\right]$. Recall, that abelian schemes satisfy a stronger Néron mapping property, cf. [10, Sec. 3.1.5]. Thus, the endomorphism $\varepsilon^{\prime}$ extends to an endomorphism

$$
\varepsilon^{\prime}: \operatorname{Pic}_{\mathcal{Y} / U}^{0} \longrightarrow \mathcal{P}_{d} \subset \operatorname{Pic}_{\mathcal{Y} / U}^{0} .
$$

Let $[T] \in \operatorname{Corr}_{U}(\mathcal{Y})$ be the class of a correspondence $T$ on $\mathcal{Y} \times_{U} \mathcal{Y}$ associated to the endomorphism $\varepsilon^{\prime}$, cf. (2.2). We write $T=\sum n_{i} T_{i}$, where $T_{i}$ are prime divisors. Let $\Sigma$ be a general two dimensional linear system in $|\mathcal{H}|_{x}$, i.e. the general member of $\Sigma$ is smooth and intersects the branch locus $\Delta$ transversally. Then, the correspondences $T_{i}$ are all defined over a non-empty open subset of $\Sigma$ and we can construct a rational map $\phi_{\Sigma, T_{i}}: S^{\prime}--\rightarrow \operatorname{Div}^{+}\left(S^{\prime}\right), y \mapsto \Gamma_{y}^{i}$ , cf. [3, pp. 38]. Especially, we get a rational map

$$
\phi_{\Sigma, T}: S^{\prime}--\rightarrow \operatorname{Pic}\left(S^{\prime}\right), y \mapsto\left[\Gamma_{y}\right]:=\sum n_{i}\left[\Gamma_{y}^{i}\right] .
$$


Let $[C] \in|\mathcal{H}|_{x}$ be a general member and choose a general two-dimensional linear system $\Sigma$ containing $[C]$. Consider the rational map $\phi_{\Sigma, T}$. Then, for a general point $y \in C^{\prime}$ we get a divisor $\Gamma_{y}=\phi_{\Sigma, T}(y)$ on $S^{\prime}$. Set $w=f(y) \in C, f^{-1}(w)=\left\{y, \sigma(y), \ldots, \sigma^{n-1}(y)\right\}$ and $f^{-1}(x)=\left\{z, \sigma(z), \ldots, \sigma^{n-1}(z)\right\}$, where $\sigma$ is a generator of the Galois group of the covering $f$. The following lemma computes the divisor $E_{y}$ in $C^{\prime}$ corresponding to the intersection of $C^{\prime}$ with $\Gamma_{y}$.

Lemma 4.1 We have that $E_{y}=\alpha_{0} z+\alpha_{1} \sigma(z)+\ldots+\alpha_{n-1} \sigma^{n-1}(z)+\beta_{0} y+\beta_{1} \sigma(y)+\ldots+$ $\beta_{n-1} \sigma^{n-1}(y)+\gamma \mathcal{B}_{x, w}^{\prime}+T_{C^{\prime}}(y)$, where $\alpha_{i}, \beta_{i}, \gamma \in \mathbb{Z}$ and $\mathcal{B}_{x, w}^{\prime}$ is the pull-back of the divisor of base points different from $x$ and $w$ of $\Sigma_{w}$ under the covering $f$.

Proof Cf. [3, Lem. 3.6].

\subsection{Regular case}

The branched locus $\Delta$ of the covering $f$ is a smooth ample divisor and thus, the canonical map $\operatorname{Alb}(f): \operatorname{Alb}\left(S^{\prime}\right) \longrightarrow \operatorname{Alb}(S)$ induced by $f$ is an isomorphism. Indeed, since $f_{*} \mathcal{O}_{S^{\prime}} \cong$ $\bigoplus_{i=0}^{n-1} \mathcal{L}^{-i}$, the Kodaira Vanishing theorem gives $H^{1}\left(\mathcal{O}_{S^{\prime}}\right)=H^{1}\left(\mathcal{O}_{S}\right)$ and hence, $\operatorname{Alb}(f)$ is an isogeny. From this one immediately sees that the induced action on $\operatorname{Alb}\left(S^{\prime}\right)$ is trivial, i.e. $\operatorname{Alb}(\sigma)=$ id. Consider the Albanese map $\operatorname{Alb}_{\xi_{o}}: S^{\prime} \longrightarrow \operatorname{Alb}\left(S^{\prime}\right)$, where the point $\xi_{o} \in S^{\prime}$ lies over a point of the branch locus $\Delta \subset S$ and observe that the map is invariant under the $\mu_{n}$-action. Therefore, we find a homomorphism $\operatorname{Alb}(S) \longrightarrow \operatorname{Alb}\left(S^{\prime}\right)$ that is inverse to $\operatorname{Alb}(f)$, proving the claim. In particular, we deduce that $q(S)=q\left(S^{\prime}\right)$. Here, we give the proof for the case $S$ is regular, i.e. $q(S)=0$.

Proof of Theorem 1.1 for the regular case If $S$ is regular, then $\operatorname{Pic}\left(S^{\prime}\right)$ is discrete and thus, the rational map $\phi_{\Sigma, T}$ is constant. Hence, for a general point $y \in C^{\prime}$, the curves $\Gamma_{y}$ and $\Gamma_{\sigma(y)}$ are linearly equivalent. It follows that $E_{y}$ and $E_{\sigma(y)}$ are also linearly equivalent and so, $E_{y}-E_{\sigma(y)}=\beta_{0}(y-\sigma(y))+\beta_{1} \sigma(y-\sigma(y))+\ldots+\beta_{n-1} \sigma^{n-1}(y-\sigma(y))+T_{C^{\prime}}(y-$ $\sigma(y)) \sim 0$. Since $\operatorname{Prym}\left(C^{\prime} / C\right)=\operatorname{im}\left(\mathrm{id}-\sigma^{*}\right)$, the latter forces $T_{C^{\prime}}(y)=\left(-\beta_{0}\right) y+\ldots+$ $\left(-\beta_{n-1}\right) \sigma^{n-1}(y)$ for all $y \in \operatorname{Prym}\left(C^{\prime} / C\right)$. Eventually, we see that the restriction of $T_{C^{\prime}}$ to $\mathcal{P}_{d}\left(C^{\prime} / C\right)$ takes the desired form. This yields that the restriction of $\varepsilon^{\prime}$ to $\left(\mathcal{P}_{d}\right)_{\eta}$ lies in $\mathbb{Z}\left[\zeta_{d}\right]$, as claimed.

\subsection{Irregular case}

The closed embedding $i: C^{\prime} \hookrightarrow S^{\prime}$ defines the natural map $i^{*}: \operatorname{Pic}^{0}\left(S^{\prime}\right) \longrightarrow \operatorname{Pic}^{0}\left(C^{\prime}\right)$ whose kernel is finite, since $H^{1}\left(S^{\prime}, \mathcal{O}_{S^{\prime}}\left(-C^{\prime}\right)\right)=0$. In what follows we view $\operatorname{Pic}^{0}\left(S^{\prime}\right)$ as an abelian subvariety of $\operatorname{Jac}\left(C^{\prime}\right)$ by identifying it with $\operatorname{im}\left(i^{*}\right)$. We shall use the following lemma.

Lemma 4.2 Let $a: \operatorname{Jac}\left(C^{\prime}\right) \longrightarrow \mathcal{P}_{d}\left(C^{\prime} / C\right) \subset \operatorname{Jac}\left(C^{\prime}\right)$ be a homomorphism and let $T_{a}$ be a correspondence associated to it, $c f$. (2.2). Assume that there exist $\alpha_{0}, \ldots, \alpha_{n-1} \in \mathbb{Z}$, such that for general $y \in C^{\prime}$ the divisor class $T_{a}(y-\sigma(y))+\alpha_{0}(y-\sigma(y))+\ldots+\alpha_{n-1} \sigma^{n-1}(y-\sigma(y))$ lies in $\mathrm{Pic}^{0}\left(S^{\prime}\right)$. Then, the restriction of a to $\mathcal{P}_{d}\left(C^{\prime} / C\right)$ lies in $\mathbb{Z}\left[\zeta_{d}\right] \subset \operatorname{End}\left(\mathcal{P}_{d}\left(C^{\prime} / C\right)\right)$.

Proof Recall that $\operatorname{Prym}\left(C^{\prime} / C\right)=\mathrm{im}\left(\mathrm{id}-\sigma^{*}\right)$ and for this reason the closed points of $\operatorname{Prym}\left(C^{\prime} / C\right)$ are generated by elements of the form $y-\sigma(y)$, where $y \in C^{\prime}$. Hence, the assumption clearly implies that $\eta(y):=a(y)+\alpha_{0} y+\ldots+\alpha_{n-1} \sigma^{n-1}(y) \in \operatorname{im}\left(i^{*}\right) \cap$ 
$\operatorname{Prym}\left(C^{\prime} / C\right)$ (note that $\mathcal{P}_{d}\left(C^{\prime} / C\right) \subset \operatorname{Prym}\left(C^{\prime} / C\right)$ ) for all $y \in \operatorname{Prym}\left(C^{\prime} / C\right)$, where $i^{*}: \operatorname{Pic}^{0}\left(S^{\prime}\right) \longrightarrow \operatorname{Pic}^{0}\left(C^{\prime}\right)=\operatorname{Jac}\left(C^{\prime}\right)$ is the natural pull-back induced by $C^{\prime} \hookrightarrow S^{\prime}$. We show that the intersection $\operatorname{im}\left(i^{*}\right) \cap \operatorname{Prym}\left(C^{\prime} / C\right)$ is finite. Indeed, consider the commutative square:

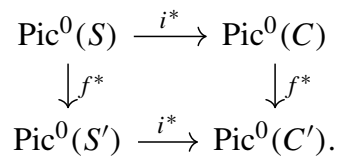

The canonical map $\operatorname{Alb}\left(S^{\prime}\right) \longrightarrow \operatorname{Alb}(S)$ induced by $f$ is an isomorphism and so, is its dual, which is $f^{*}$. Hence, the latter yields that $\operatorname{im}\left(i^{*}: \operatorname{Pic}^{0}\left(S^{\prime}\right) \longrightarrow \operatorname{Pic}^{0}\left(C^{\prime}\right)\right) \subset f^{*}\left(\operatorname{Pic}^{0}(C)\right)$. By the definition of $\operatorname{Prym}\left(C^{\prime} / C\right)$, we know that $f^{*}\left(\operatorname{Pic}^{0}(C)\right) \cap \operatorname{Prym}\left(C^{\prime} / C\right)$ is finite and so, is the intersection $\operatorname{im}\left(i^{*}\right) \cap \operatorname{Prym}\left(C^{\prime} / C\right)$, as claimed. From the latter one deduces that the endomorphism $\eta$ of $\operatorname{Prym}\left(C^{\prime} / C\right)$ defined above is the zero-map, simply because $\eta\left(\operatorname{Prym}\left(C^{\prime} / C\right)\right)$ is irreducible subvariety of $\operatorname{im}\left(i^{*}\right) \cap \operatorname{Prym}\left(C^{\prime} / C\right)$, which is a finite union of points. Finally, by restricting to $\mathcal{P}_{d}\left(C^{\prime} / C\right) \subset \operatorname{Prym}\left(C^{\prime} / C\right)$, we conclude that $a$ lies in the image of the map $\mathbb{Z}\left[\zeta_{d}\right] \subset \operatorname{End}\left(\mathcal{P}_{d}\left(C^{\prime} / C\right)\right), \zeta_{d} \mapsto \sigma$. The proof is complete.

Proof of Theorem 1.1 for the irregular case Using the curves $\Gamma_{y}$ we find that $E_{y}-E_{\sigma(y)}$ lies in the image of $\operatorname{Pic}\left(S^{\prime}\right) \longrightarrow \operatorname{Pic}\left(C^{\prime}\right)$. Therefore, we have that $T_{C^{\prime}}(y-\sigma(y))+\beta_{0}(y-\sigma(y))+$ $\beta_{1} \sigma(y-\sigma(y))+\ldots+\beta_{n-1} \sigma^{n-1}(y-\sigma(y)) \in \operatorname{im}\left(i^{*}: \operatorname{Pic}\left(S^{\prime}\right) \longrightarrow \operatorname{Pic}\left(C^{\prime}\right)\right)$ for general $y \in C^{\prime}$. It follows that $\varepsilon^{\prime} \in \mathbb{Z}\left[\zeta_{d}\right] \subset \operatorname{End}\left(\left(\mathcal{P}_{d}\right)_{\eta}\right)$, cf. (4.2).

\section{The proof of Theorem 1.3}

The proof is similar to the case of (1.1). First, we need to replace our earlier family $\varphi_{d}: \mathcal{P}_{d} \longrightarrow U$. In particular, we consider the abelian fibration

$$
\mathcal{R}_{d}:=\operatorname{ker}^{0}\left(\mathcal{P}_{d} \longrightarrow \operatorname{Alb}\left(S^{\prime}\right) \times U\right) .
$$

Assume that the abelian fibration $\varphi_{d}: \mathcal{R}_{d} \longrightarrow U$ is non-zero, i.e. $\mathcal{R}_{[C]} \neq 0$ for $[C] \in U$. Then, we show that for the very general member $[C] \in U$, we have that $\operatorname{End}_{\mu_{d}}\left(\left(\mathcal{R}_{d}\right)_{[C]}\right) \cong$ $\mathbb{Z}\left[\zeta_{d}\right]$. One checks that the results (3.3) and (3.4) still hold true for the family $\varphi_{d}: \mathcal{R}_{d} \longrightarrow U$.

We proceed as in the proof of Theorem 1.1. A non-zero endomorphism $\varepsilon \in \operatorname{End}_{\mu_{d}}\left(\left(\mathcal{R}_{d}\right)_{\eta}\right)$ gives rise to an endomorphism $\varepsilon^{\prime} \in \operatorname{End}\left(\operatorname{Jac}\left(C_{\eta}^{\prime}\right)\right)$ and it is enough to check that the restriction of $\varepsilon^{\prime}$ to $\left(\mathcal{R}_{d}\right)_{\eta}$ lies in $\mathbb{Z}\left[\zeta_{d}\right]$. The following lemma is needed.

Lemma 5.1 Let $a: \operatorname{Jac}\left(C^{\prime}\right) \longrightarrow \mathcal{R}_{d}\left(C^{\prime}, C, S^{\prime}\right) \subset \operatorname{Jac}\left(C^{\prime}\right)$ be a homomorphism and let $T_{a}$ be a correspondence associated to it, $c f$. (2.2). Assume that there exist $\alpha_{0}, \ldots, \alpha_{n-1} \in \mathbb{Z}$, such that for general $y \in C^{\prime}$ the divisor class $T_{a}(y-\sigma(y))+\alpha_{0}(y-\sigma(y))+\ldots+$ $\alpha_{n-1} \sigma^{n-1}(y-\sigma(y))$ lies in $\mathrm{Pic}^{0}\left(S^{\prime}\right)$. Then, the restriction of a to $\mathcal{R}_{d}\left(C^{\prime}, C, S^{\prime}\right)$ lies in $\mathbb{Z}\left[\zeta_{d}\right] \subset \operatorname{End}\left(\mathcal{R}_{d}\left(C^{\prime}, C, S^{\prime}\right)\right)$.

Proof Clearly, we have that $a(y)+\alpha_{0} y+\ldots+\alpha_{n-1} \sigma^{n-1}(y) \in \operatorname{im}\left(i^{*}\right)$ for all $y \in$ $\operatorname{Prym}\left(C^{\prime} / C\right)$, where $i^{*}: \operatorname{Pic}^{0}\left(S^{\prime}\right) \longrightarrow \operatorname{Pic}^{0}\left(C^{\prime}\right)=\operatorname{Jac}\left(C^{\prime}\right)$ is the pull-back induced by $C^{\prime} \hookrightarrow S^{\prime}$. Let $\mathcal{K}\left(C^{\prime}, S^{\prime}\right):=\operatorname{ker}\left(\operatorname{Jac}\left(C^{\prime}\right) \longrightarrow \operatorname{Alb}\left(S^{\prime}\right)\right)$ and observe that the intersection $\operatorname{im}\left(i^{*}\right) \cap \mathcal{K}\left(C^{\prime}, S^{\prime}\right)$ is finite. Since $\mathcal{R}_{d}\left(C^{\prime}, C, S^{\prime}\right) \subset \mathcal{K}\left(C^{\prime}, S^{\prime}\right)$, we find that $a(y)+\alpha_{0} y+\ldots+\alpha_{n-1} \sigma^{n-1}(y)=0$ for all $y \in \mathcal{R}_{d}\left(C^{\prime}, C, S^{\prime}\right)$. Therefore, the restriction of $a$ to $\mathcal{R}_{d}\left(C^{\prime}, C, S^{\prime}\right)$ belongs to $\mathbb{Z}\left[\zeta_{d}\right]$, as claimed. 
Proof of Theorem 1.3 Using the curves $\Gamma_{y}$ one sees that $E_{y}-E_{\sigma(y)}$ lies in the image of $\operatorname{Pic}\left(S^{\prime}\right) \longrightarrow \operatorname{Pic}\left(C^{\prime}\right)$. It follows that $T_{C^{\prime}}(y-\sigma(y))+\beta_{0}(y-\sigma(y))+\beta_{1} \sigma(y-\sigma(y))+$ $\ldots+\beta_{n-1} \sigma^{n-1}(y-\sigma(y)) \in \operatorname{im}\left(i^{*}: \operatorname{Pic}\left(S^{\prime}\right) \longrightarrow \operatorname{Pic}\left(C^{\prime}\right)\right)$. Now, the result is an immediate consequence of (5.1).

Acknowledgements The author thanks his advisor Professor Dr. Daniel Huybrechts and Dr. Gebhard Martin for helpful discussions on the topics of this note, which are parts of the author's Master thesis, as well as for corrections.

Funding Open Access funding enabled and organized by Project DEAL.

Data availibility statement Not applicable.

Open Access This article is licensed under a Creative Commons Attribution 4.0 International License, which permits use, sharing, adaptation, distribution and reproduction in any medium or format, as long as you give appropriate credit to the original author(s) and the source, provide a link to the Creative Commons licence, and indicate if changes were made. The images or other third party material in this article are included in the article's Creative Commons licence, unless indicated otherwise in a credit line to the material. If material is not included in the article's Creative Commons licence and your intended use is not permitted by statutory regulation or exceeds the permitted use, you will need to obtain permission directly from the copyright holder. To view a copy of this licence, visit http://creativecommons.org/licenses/by/4.0/.

\section{References}

1. Antei, M.: On the abelian fundamental group scheme of a family of varieties. English. In: Isr. J. Math. 186, 427-446. issn: 0021-2172. https://doi.org/10.1007/s11856-011-0147-9 (2011)

2. Carocca, A., Rodríguez, R.: Jacobians with group actions and rational idempotents. English. J. Algebra 306(2) , 322-343. issn: 0021-8693. https://doi.org/10.1016/j.jalgebra.2006.07.027. (2006)

3. Ciliberto, C., van der Geer, G.: On the Jacobian of a hyperplane section of a surface. English. In: Classification of Irregular Varieties. Minimal Models and Abelian Varieties. Proceedings of a Conference, Held in Trento, Italy, 17-21 (1990). Berlin etc.: Springer, pp. 33-40. isbn: 3-540-55295 (1992)

4. Fantechi, B., et al.: Fundamental algebraic geometry: Grothendieck's FGA explained. English. Vol. 123. Providence, RI: American Mathematical Society (AMS), pp. $x+339$. isbn: 0-8218-3541-6. (2005)

5. Kleiman, S.L.: The Picard scheme (2005) arXiv: math/0504020 [math.AG]

6. Lange, H., Ortega, A.: The fibers of the Prym map of étale cyclic coverings of degree 7. In: Contemporary Mathematics Contributions of Mexican Mathematicians Abroad in Pure and Applied Mathematics, pp. 75-85 (2018). https://doi.org/10.1090/conm/709/14293

7. Lange, H., Pishmish, S.R.: Abelian varieties with group action. English. In: Reine Angew R. Math. 575 , 135-155. issn: 0075-4102 (2004). https://doi.org/10.1515/crll.2004.076

8. Laurent, B., Schröer, S.: Para-abelian varieties and Albanese maps. arXiv: 2101.10829, [math.AG] (2021)

9. Milne, J.S.: Abelian varieties. In: Arithmetic Geometry. Ed. by Gary Cornell and Joseph H. Silverman. New York, NY: Springer, pp. 103-150. ISBN: 978-1-4613-8655-1 (1986)

10. Robert, D.: Theory of abelian varieties and their moduli spaces, lecture notes (2021). https://www. normalesup.org/ robert/pro/publications/books/avtheory.pdf

11. Rojas, A.M.: Group actions on Jacobian varieties. English. Rev. Mat. Iberoam. 23(2), 397-420. ISBN: 0213-2230 (2007). https://doi.org/10.4171/RMI/500

12. Raynaud, M.S.B.W.L.: Néron Models, ISBN:, 1st 21., pp. 978-3-540-50587-7. Springer, Berlin Heidelberg (1990)

13. edu.: The Stacks Project Authors. Stacks Project (2021)

14. Görtz, T., Wedhorn, U.: Algebraic Geometry I. Vieweg+Teubner. ISBN, 978-3-8348-0676-5 (2010)

Publisher's Note Springer Nature remains neutral with regard to jurisdictional claims in published maps and institutional affiliations. 